\title{
THE LARGEST PROPER VARIETY OF LATTICE ORDERED GROUPS
}

\author{
W. CHARLES HOLLAND
}

\begin{abstract}
If a lattice ordered group $G$ satisfies any identical relation, other than those satisfied by every lattice ordered group, then $G$ is normal valued, and hence satisfies the relation $a b \leqslant b^{2} a^{2}$ for all $a, b \geqslant e$.
\end{abstract}

A lattice ordered group ( $l$-group) $G$ is said to be normal valued if whenever $M$ is a convex $l$-subgroup maximal with respect to missing a fixed element $g \in G$, and $K$ the smallest convex $l$-subgroup of $G$ containing $M$ and $g$, then $M$ is a normal subgroup of $K$. It was shown by Wolfenstein [5] that the normal valued $l$-groups are characterized by the property that $a b \leqslant b^{2} a^{2}$ for all $a, b \geqslant e$, and thus constitute a variety, or equationally defined class (the inequality is equivalent to the equation $|x||y||x|^{-2}|y|^{-2} \vee e=e$, where $\left.|z|=\left(z \vee z^{-1}\right)\right)$. It has been observed that the variety of normal valued $l$ groups is very large; all of the many varieties studied by Martinez [2] are contained in the normal valued variety (with the exception of the variety of all l-groups). It will be shown here that every property variety of lattice ordered groups is contained in the normal valued variety. This sheds a new light on several of Martinez's results, and shows that certain l-groups are generic. For example, if $A(\mathbf{R})$ denotes the $l$-group of all order preserving permutations of the real line, and if $A(\mathbf{R})$ satisfies an identical relation, then every l-group must satisfy that relation.

THEOREM. If a lattice ordered group $G$ satisfies an identical relation which is not satisfied by every lattice ordered group, then $G$ is normal valued.

The Theorem will be proved in a sequence of lemmas.

If an $l$-group $H$ is an $l$-subgroup of the $l$-group $A(S)$ of all order preserving permutations of a totally ordered set $S, H$ is said to be $o$-2-transitive on $S$ if whenever $s_{1}<s_{2}, t_{1}<t_{2}$ are members of $S$, there exists $h \in H$ such that $s_{i} h=t_{i}$. It follows easily that any such $H$ must in fact be o- $n$-transitive in the sense that whenever $s_{1}<s_{2}<\cdots<s_{n}, t_{1}<t_{2}<\cdots<t_{n}$ are members of $S$, there exists $h \in H$ such that $s_{i} h=t_{i}$ [4, Lemma 4]. Again, $H$ is said to be o-primitive on $S$ if $H$ acts transitively on $S$ and the stabilizer subgroups $H_{s}=\{h \in H \mid s h=s\}$ are maximal convex $l$-subgroups. Finally, $H$ is periodic on $S$ if $H$ is transitive on $S$ and there is a period $f \in A(\bar{S})$, where $\bar{S}$ is the Dedekind completion of $S$, such that $f h=h f$ for all $h \in H$, where $H \subseteq A(\bar{S})$

Received by the editors January 27, 1975.

AMS (MOS) subject classifications (1970). Primary 06A55.

Key words and phrases. Lattice ordered group, variety, normal valued.

- American Mathematical Society 1976 
in the natural way, and $f$ has coterminal orbits. It was shown by McCleary [3] that if $H$ is o-primitive on $S$ then either $H_{s}=\{e\}$ for every $s \in S$, or $H$ is o-2transitive on $S$, or $H$ is periodic on $S$. In the latter case, $H_{s}$ acts faithfully and o-2-transitively on the interval $(s, s f)$ of $S$, where $f$ is the period, and $s$ is any member of $S$.

LEMMA 1. If the l-subgroup $H$ of $A(S)$ is o-primitive on $S$ but $H_{s} \neq\{e\}$ for some $s \in S$, then $H$ contains an l-subgroup which is o-2-transitive on some totally ordered set.

Now suppose that $G$ is an $l$-group which is not normal valued. There exists, then, a convex $l$-subgroup $M$ of $G$, maximal with respect to missing some element $g \in G$, such that $M$ is not a normal subgroup of its cover $K$. The intersection $\cap k^{-1} M k$ of all the conjugates of $M$ in $K$ is an $l$-ideal of $K$, and the $l$-group $H=K / \cap k^{-1} M k$ is $l$-isomorphic to an o-primitive $l$-subgroup of order preserving permutations of the totally ordered set $S$ of right cosets of $M$ in $K$. In this representation, $H_{s}=M$ for some $s \in S$, and so $H_{s} \neq\{e\}$ since $M$ is not normal in $K$. By Lemma $1, H$ contains an $l$-subgroup which is o-2transitive on some set. This subgroup must belong to any variety that contains $G$. Thus:

LEMMA 2. If $G$ is an l-group which is not normal valued, then every variety containing $G$ contains an l-group which is an o-2-transitive l-group of order preserving permutations of some totally ordered set.

Let $X$ be a countably infinite set of letters and $X^{-1}=\left\{x^{-1} \mid x \in X\right\}$ a set disjoint from $X$. Let $F$ be the free $l$-group on $X$. The elements of $F$ may be written in the form $\vee_{A} \wedge_{B} \Pi_{\Gamma} x_{\alpha \beta \gamma}$ where $A, B, \Gamma$ are finite index sets, $\Gamma=\{1,2, \ldots, n\}, x_{\alpha \beta \gamma} \in X \cup X^{-1} \cup\{e\}, \Pi$ indicates the group operation, and $\vee$ and $\wedge$ the lattice operations. There is, in general, nothing unique about the form of a given element of $F$. An identical relation is, then, a formal expression $w=e$, where $w \in F$. An $l$-group $H$ is said to satisfy the identical relation $w=e$, where $w$ has the form above, if for every substitution $x_{\alpha \beta \gamma} \mapsto h_{\alpha \beta \gamma}$ by elements of $H$, we have $e=\bigvee_{A} \wedge_{B} \Pi_{\Gamma} h_{\alpha \beta \gamma}$, where it is understood that if $h$ is substituted for one occurrence of $x, h$ must also be substituted for all other occurrences of the same $x, h^{-1}$ for $x^{-1}$, and $e$ (in $H$ ) for $e$ (in $F$ ), and $\vee, \wedge, \Pi$ indicate the lattice and group operations in $H$.

LEMMA 3. Let $H$ be a nontrivial o-2-transitive l-group of order preserving permutations of a totally ordered set $S$, and $w \in F$ not the identity element of the free l-group $F$. Then $H$ does not satisfy the identical relation $w=e$.

To prove Lemma 3, it may first be assumed that $F$ is an l-group of order preserving permutations of a totally ordered set $T$ [1]. There must exist a point $t \in T$ such that $t w \neq t$. Let $w=\vee_{A} \wedge_{B} \Pi_{\Gamma} x_{\alpha \beta \gamma}, \Gamma=\{1,2, \ldots, n\}$. For each $(\alpha, \beta) \in A \times B$, define $t(\alpha, \beta, 0)=t$, and for $1 \leqslant \gamma \leqslant n, t(\alpha, \beta, \gamma)$ $=t(\alpha, \beta, \gamma-1) x_{\alpha \beta \gamma}$. Now for each $x \in X$ occurring in $w$, and each $(\alpha, \beta)$, let $P_{\alpha \beta}(x)=\left\{\gamma \mid x=x_{\alpha \beta \gamma}\right\}$ and $N_{\alpha \beta}(x)=\left\{\gamma \mid x^{-1}=x_{\alpha \beta \gamma}\right\}$. Then if $\gamma \in P_{\alpha \beta}(x)$, $t(\alpha, \beta, \gamma-1) x=t(\alpha, \beta, \gamma)$, while if $\gamma \in N_{\alpha \beta}(x), t(\alpha, \beta, \gamma) x=t(\alpha, \beta, \gamma-1)$.

The set $T^{\prime}=\{t(\alpha, \beta, \gamma) \mid(\alpha, \beta) \in A \times B, 0 \leqslant \gamma \leqslant n\}$ is a finite subset of $T$. Now choose and label any subset $\{s(\alpha, \beta, \gamma) \mid(\alpha, \beta) \in A \times B, 0 \leqslant \gamma \leqslant n\}$ of $S$ 
in one-to-one correspondence with $T^{\prime}$, so that the correspondence $t(\alpha, \beta, \gamma)$ $\leftrightarrow s(\alpha, \beta, \gamma)$ preserves order. Since multiplication by $x$ provides a one-to-one order preserving correspondence such that

$$
\begin{aligned}
t(\alpha, \beta, \gamma-1) & \mapsto t(\alpha, \beta, \gamma) \quad \text { for } \gamma \in P_{\alpha \beta}(x), \\
t(\alpha, \beta, \gamma) & \mapsto t(\alpha, \beta, \gamma-1) \text { for } \gamma \in N_{\alpha \beta}(x),
\end{aligned}
$$

it follows that the correspondence

$$
\begin{aligned}
s(\alpha, \beta, \gamma-1) & \mapsto s(\alpha, \beta, \gamma) \quad \text { for } \gamma \in P_{\alpha \beta}(x), \\
s(\alpha, \beta, \gamma) & \mapsto s(\alpha, \beta, \gamma-1) \text { for } \gamma \in N_{\alpha \beta}(x)
\end{aligned}
$$

must also be one-to-one and order preserving. As $H$ is o-2-transitive on $S, H$ is also o- $n$-transitive, and hence there exists $h(x) \in H$ such that

$$
\begin{aligned}
s(\alpha, \beta, \gamma-1) h(x) & =s(\alpha, \beta, \gamma) \quad \text { for } \gamma \in P_{\alpha \beta}(x), \\
s(\alpha, \beta, \gamma) h(x) & =s(\alpha, \beta, \gamma-1) \text { for } \gamma \in N_{\alpha \beta}(x) .
\end{aligned}
$$

Since $t=t(\alpha, \beta, 0)$ for each $(\alpha, \beta)$, we may let $s=s(\alpha, \beta, 0)$. Then substituting $x \mapsto h(x)$ (and $\left.x^{-1} \mapsto h\left(x^{-1}\right)=(h(x))^{-1}, e \mapsto e\right)$, we have for each $(\alpha, \beta)$ $\in A \times B$,

$$
s \Pi_{\Gamma} h\left(x_{\alpha \beta \gamma}\right)=s(\alpha, \beta, n)
$$

Since $t w \neq t$,

$$
t \neq t \vee_{A} \wedge_{B} \Pi_{\Gamma} x_{\alpha \beta \gamma}=\vee_{A} \wedge_{B} t \Pi_{\Gamma} x_{\alpha \beta \gamma}=\vee_{A} \wedge_{B} t(\alpha, \beta, n),
$$

where on the right side of the equation, the lattice operations are taken on the finite chain $\{t(\alpha, \beta, n)\}$, which is in one-to-one order preserving correspondence with the chain $\{s(\alpha, \beta, n)\}$. It follows that

$$
s \vee_{A} \wedge_{B} \Pi_{\Gamma} h\left(x_{\alpha \beta \gamma}\right)=\vee_{A} \wedge_{B} s \Pi_{\Gamma} h\left(x_{\alpha \beta \gamma}\right)=\vee_{A} \wedge_{B} s(\alpha, \beta n) \neq s .
$$

Hence $\vee_{A} \wedge_{B} \Pi_{\Gamma} h\left(x_{\alpha \beta \gamma}\right) \neq e$ in $H$, and $H$ fails to satisfy the identical relation $w=e$, proving Lemma 3.

Now to prove the Theorem, let $G$ be an $l$-group which is not normal valued and let $w=e$ be an identical relation which is not satisfied by every $l$-group. By Lemma 2 , any variety containing $G$ must contain an $l$-group $H$ which is o-2-transitive on some totally ordered set. By Lemma 3, $H$ fails to satisfy $w=$ $e$, and hence so does $G$.

COROLLARY. The variety of normal valued l-groups is the largest proper variety of l-groups and contains every other proper variety of l-groups.

In [2], Martinez showed that the variety $\mathfrak{L}$ of all $l$-groups is finitely join irreducible in the lattice of varieties of $l$-groups. But a much stronger statement is now obvious.

COROLlary. The variety $\mathcal{L}$ of all l-groups is completely join irreducible in the lattice of varieties of l-groups. 
COROLLARY (MARTINEZ [2]). The variety of normal valued l-groups is idempotent.

For a proof, it suffices to produce an l-group which is not an extension of a normal valued $l$-group by a normal valued $l$-group. The $l$-group $B(\mathbf{R})$ of all order-preserving permutations of the real line $\mathbf{R}$ having bounded support is $l$ simple [1] and not normal valued, and so serves this purpose.

The free $l$-group on a countable set is generic in the sense that it generates the variety of all $l$-groups. A much more tractable example is given by

CoROllaRy (To Lemma 3). The l-group A(R) of all order-preserving permutations of the real line is generic-if $A(\mathbf{R})$ satisfies a certain identical relation, every l-group satisfies that relation.

\section{REFERENCES}

1. C. Holland, The lattice-ordered group of automorphisms of an ordered set, Michigan Math. J. 10 (1963), 399-408. MR 28 \#1237.

2. J. Martinez, Varieties of lattice-ordered groups, Math. Z. 137 (1974), 265-284.

3. S. H. McCleary, o-primitive ordered permutation groups. I, II, Pacific J. Math. 40 (1972), 349-372; 49 (1973), 431-443. MR 47 \#1710.

4. - o-2-transitive ordered permutation groups, Pacific J. Math. 49 (1973), 425-429.

5. S. Wolfenstein, Valeurs normales dans un groupe réticulé, Atti Accad. Naz. Lincei Rend. Cl. Sci. Fis. Mat. Natur. (8) 44 (1968), 337-342. MR 38 \#3201.

Department of Mathematics, Bowling Green State University, Bowling Green, Ohio 43403 